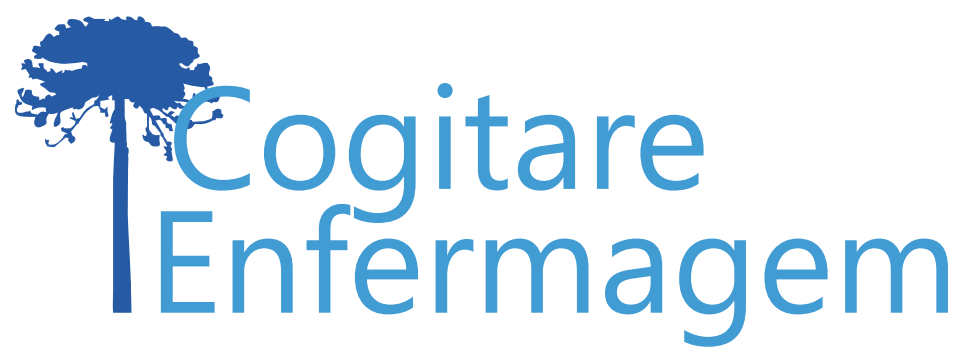

\title{
DEZ ANOS DA RESIDÊNCIA MULTIPROFISSIONAL DO COMPLEXO HOSPITAL DE CLÍNICAS DA UNIVERSIDADE FEDERAL DO PARANÁ
}

\section{TEN YEARS OF THE MULTIPROFESSIONAL RESIDENCY OF THE CLINICAL HOSPITAL COMPLEX OF THE FEDERAL UNIVERSITY OF PARANÁ}

\author{
Liane Fuhr Pivatto ${ }^{1}$ (1) \\ Vânia Mari Salvi Andrzejevski ${ }^{1}$ (1) \\ Rita Aparecida Bernardi Pereira ${ }^{1}$ (i) \\ Tatiana Brusamarello ${ }^{1}$ (1) \\ Jorge Vinícius Cestari Félix
}

\begin{abstract}
Objective: to report the experience of the ten years since the implementation of the Multiprofessional Residency Program in Hospital Care at the Clinical Hospital Complex of the Federal University of Paraná. Development: an experience report on the ten years since the implementation of the residency program. The data were collected from internal documents and from the coordinators' and preceptors' reports. Three hundred and seventy-two professionals were trained. Among the advances of the program, the increase in the number of vacancies and specialties, consolidation of the Internal Regulations, computerized management of the Academic information, incentive to research, and improvement of the assistance provided in the hospital environment stand out. Final Considerations: the ten years of the program were marked by the challenge of deconstructing the uniprofessional perspective towards a project that contemplated the diversity of the multiple professions, providing the construction of new paradigms, transformation of the professional practices, development of teamwork skills, teaching-service integration, and advancement in training.
\end{abstract}

DESCRIPTORS: Education, Nursing; Health Education; Internship and Residency; Internship, Nonmedical. do Complexo Hospital de Clínicas da Universidade Federal do Paraná. Cogit. Enferm. [Internet]. 2021 [acesso em "colocar data de acesso, dia, mês abreviado e ano"]; 26. Disponível em: http://dx.doi.org/10.5380/ce.v26i0.76053. 
A Residência Multiprofissional em Saúde (RMS) é uma modalidade de pós-graduação Lato sensu, voltada à formação e treinamento em serviço, sob supervisão docenteassistencial e em regime de dedicação exclusiva. Orientada pelos princípios e diretrizes do Sistema Único de Saúde (SUS), a partir de necessidades locais e regionais, conforme Lei $\mathrm{n}^{\circ}$ 11.129 de $2005^{(1)}$, destina-se a especializar profissionais da área da saúde não médicos ${ }^{(2)}$.

Concebida com o objetivo de propiciar transformações na prática assistencial em saúde, fomenta processo formativo alicerçado na integração e na articulação entre diferentes profissões da área da saúde ${ }^{(3-4)}$.

No entanto, vivenciar essa integração multiprofissional e interdisciplinar é um constante desafio para os docentes, coordenadores, tutores, preceptores e demais profissionais que atuam nos programas de RMS, pois requer que conflitos sejam expostos e enfrentados diariamente de forma dialógica, nos diversos cenários de prática dos residentes ${ }^{(4)}$.

Este estudo objetiva relatar a experiência dos dez anos de implementação do Programa de Residência Multiprofissional em Atenção Hospitalar (PRIMAH) no Complexo Hospital de Clínicas da Universidade Federal do Paraná (CHC-UFPR).

\section{DESENVOLVIMENTO}

Trata-se de estudo descritivo, do tipo relato de experiência, sobre o processo de implantação e consolidação do PRIMAH no CHC-UFPR. Os dados foram coletados no período de março a maio de 2020, por meio da análise de documentos e arquivos do programa e de relatos dos coordenadores e preceptores que, em sua maioria, atuam no Programa desde a sua implantação.

Nos dois anos que antecederam a sua abertura (em 2010), foram desenvolvidos intensos trabalhos e inúmeras reuniões com discussões coletivas e integradas para a elaboração do Plano Político Pedagógico (PPP) das especialidades, incluindo as disciplinas do eixo transversal (comum a todas as áreas e profissões), das áreas de concentração e das áreas profissionais. Além disso, a partir dessas discussões, também se estabeleceu a definição, a organização e a preparação dos cenários de prática, com a efetiva integração ensino-serviço.

A duração do Programa de residência é de dois anos, com carga horária de 5.760 horas, sendo $80 \%$ destas horas destinadas à atividade prática e $20 \%$ à fundamentação teórica e teórico-prática ${ }^{(1)}$. Para a integralização do curso, é obrigatória a elaboração de um Trabalho de Conclusão de Residência (T⿱T一) em formato de artigo científico e sua submissão em revista científica indexada ${ }^{(1-2)}$. Desta forma, a proposta do programa é que se contribua para a produção de conhecimento científico novo e que se fomente a constante busca por estudos e pesquisas que transformem a realidade dos serviços e propiciem o desenvolvimento de competências necessárias aos profissionais de saúde que atuam na instituição.

Em 2009, o PRIMAH finalmente abriu seu primeiro Edital de Processo Seletivo público, realizado pelo Núcleo de Concursos da UFPR, com a efetiva entrada de residentes em 01 de fevereiro de 2010. Inicialmente, foram ofertadas 21 vagas em cinco Áreas de Concentração: Atenção à Saúde do Adulto e do Idoso (PSAl), Atenção à Saúde da Mulher (SM), Oncologia e Hematologia (OH), Atenção Cardiovascular (CV) e Urgência e Emergência (UE), incluindo 8 categorias profissionais: Enfermagem, Fisioterapia, Nutrição, Serviço Social, Farmácia, 
Farmácia-Bioquímica, Psicologia e Terapia Ocupacional.

É importante ressaltar que conquistas significativas foram alcançadas nesses dez anos do PRIMAH, tais como: a ampliação do número vagas e de especialidades (Figura 1), maior incentivo à pesquisa e a melhoria da assistência à saúde no âmbito hospitalar, representando mudança significativa no dia a dia dos profissionais envolvidos com a residência.

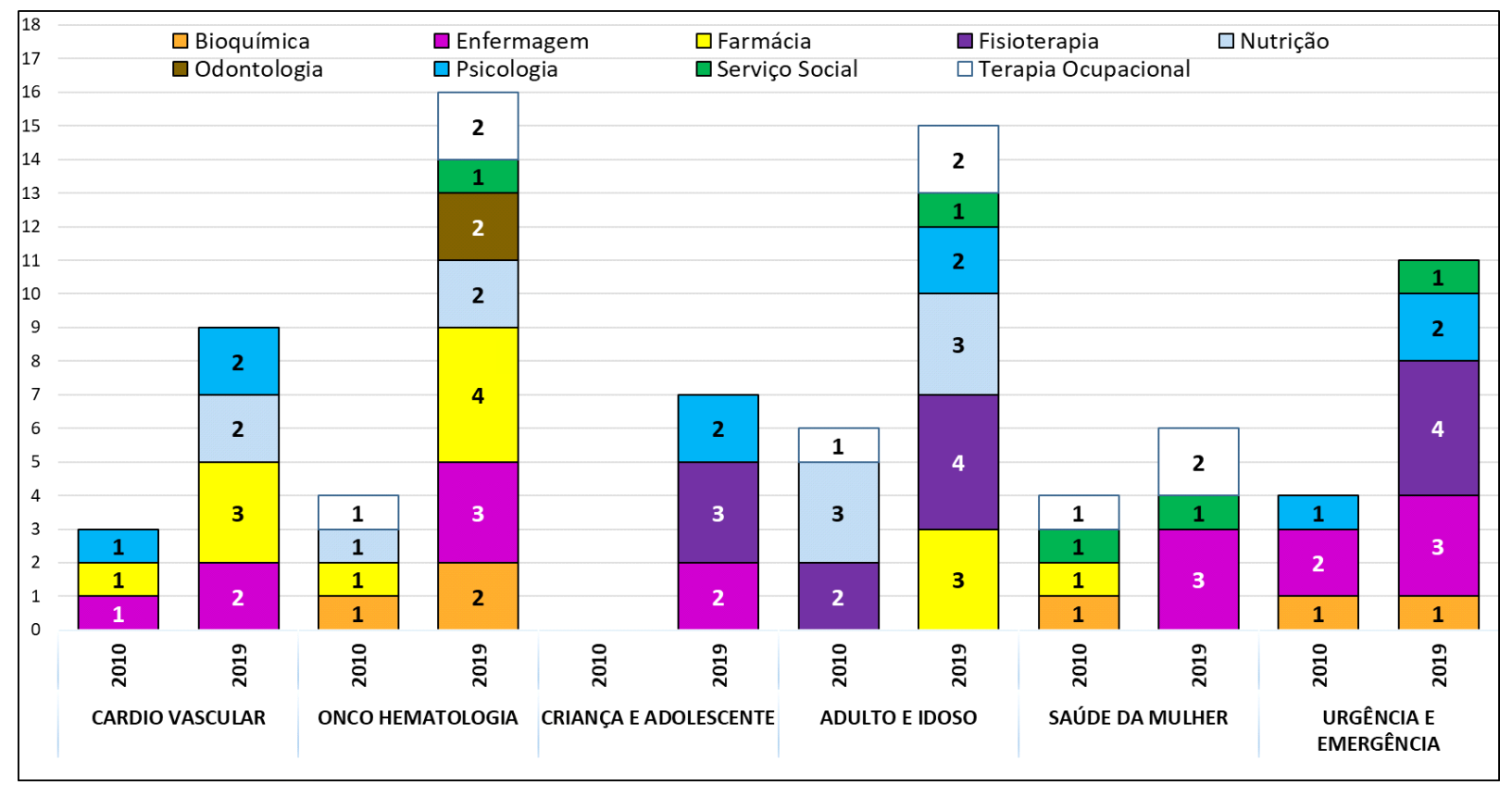

Figura 1 - Áreas de concentração e evolução das vagas ofertadas, 2010 a 2019. Curitiba, PR, Brasil, 2020 Fonte: PRIMAH-CHC-UFPR

Registram-se diversas ações para aprimorar a prática pedagógica e a supervisão dos residentes, como por exemplo a busca por aperfeiçoamento dos profissionais dos serviços, por meio de Programas de Pós-graduação Stricto e Lato sensu e de cursos de capacitação de preceptores.

Com vistas a dar visibilidade e notoriedade à produção acadêmica e científica da Residência Multiprofissional, em 2020 foi realizado o III Congresso dos Programas de Residência do CHC-UFPR com apresentação dos TCRs de todos os residentes formandos (R2), o que também oportunizou a integração entre as diferentes áreas de concentração do PRIMAH em conjunto com os Programas de Residência Médica correlatos a essas áreas.

O PRIMAH, até o presente momento, já formou 372 especialistas, tendo alcançado notoriedade frente à comunidade como referência na formação de especialistas na área da saúde. Esse fato é evidenciado pela excelente colocação profissional de seus egressos e pelo aumento expressivo observado, ano após ano, no número de inscritos no Processo Seletivo.

As diversas áreas profissionais que investiram e acreditaram na residência o fizeram procurando aproximação da prática dos serviços com o ensino e a gestão, o que, por si só, já se constitui inegável inovação na reformulação de políticas para formação de profissionais da área da saúde voltados para os princípios e diretrizes do SUS ${ }^{(1)}$. 
Desta forma, tanto o PPP do PRIMAH como a sua operacionalização se traduzem numa complexa e efetiva integração ensino-serviço, entendida como o trabalho coletivo, integrado e formalmente pactuado, realizado por residentes, docentes, tutores, preceptores, profissionais de saúde dos serviços e gestores, de forma a impactar positivamente na excelência da formação e na qualidade dos serviços ${ }^{(5-6)}$. Destaca-se a consolidação do programa na instituição por meio do desenvolvimento e publicação de seu Regimento Interno, do Calendário Acadêmico anual, do Manual do Residente e do gerenciamento informatizado das informações por meio do Sistema de Gestão Acadêmica (SIGA), plenamente integrado com a UFPR.

Inúmeros e significativos desafios ocorreram ao longo deste percurso. Dentre eles, pode-se destacar a dificuldade de comunicação entre as diferentes profissões, fragilidades na infraestrutura e até mesmo, num primeiro momento, a relativa desvalorização do programa junto à comunidade do CHC-UFPR, quando ainda não se vislumbrava a sua real importância e o impacto benéfico que teria na transformação das práticas em diferentes níveis dentro da instituição.

Desde o início, os residentes sentiam a falta de estrutura nos cenários de prática, tais como salas de aula, laboratório de informática, dentre outros espaços para construção do saber de modo multiprofissional. No entanto, reformas na instituição têm minimizado alguns problemas como melhorias nas salas de aula e a construção em andamento de um laboratório de informática e de um centro de simulação realística para treinamento dos residentes.

É importante ressaltar que o trabalho de articulação realizado pelo grupo de implantação durante esses anos assegurou a consolidação do Programa e a harmonia entre as atividades. Este grupo de profissionais (dos serviços) assume diariamente, em conjunto com suas atribuições, o compromisso de planejar, executar e avaliar todas as atividades de formação dos residentes no decorrer do programa. Destaca-se o fundamental papel dos coordenadores, gestores, tutores e preceptores do $\mathrm{CHC}$ e da UFPR para imersão dos residentes na produção do cuidado ao paciente.

Uma das grandes questões a serem enfrentadas continuamente é a escassez de profissionais no quadro assistencial em algumas profissões e serviços, o que acaba provocando sobrecarga de trabalho para os preceptores e coordenadores. Além disso, há em certa medida, dificuldade de compreensão de alguns profissionais sobre a importância da RMS, impactando diretamente na melhor integração dos residentes com os serviços. No entanto, a contratação de novos funcionários nos últimos anos, bem como a sensibilização desses profissionais para atuar na residência e a participação de docentes dos diversos departamentos do Setor de Ciências da Saúde, têm minimizado essas situações.

Contudo, é possível afirmar que instituir o PRIMAH foi um imenso e gratificante desafio, alcançado pela integração dos diversos serviços do CHC, Departamentos da UFPR e diferentes profissões e profissionais de saúde. Esssa diversidade que, a princípio, parecia ser um obstáculo intransponível, foi fundamental para a construção de um projeto pautado no respeito às diferenças, na conduta ética dos seus membros e especialmente na construção de um trabalho multiprofissional e interdisciplinar no mesmo campo de trabalho, sem deixar de priorizar e respeitar os núcleos específicos de saberes de cada profissão(5).

\section{CONSIDERAÇÕES FINAIS}

O gerenciamento da residência é um desafio permanente, visando à manutenção da qualidade do programa, ampliação de vagas, adesão de novas áreas e aprimoramento da assistência. Isso remete às coordenações constante reflexão e atualização do projeto 
pedagógico, em busca de potencialidades e políticas para transformação do modelo de atenção e das práticas de assistência multiprofissional em saúde a partir da aprendizagem vivenciada no contexto real do trabalho.

A residência configura uma importante ferramenta na construção de novos paradigmas e competências do trabalho em equipe, representando grande avanço na formação dos profissionais e melhoria da assistência. Neste sentido, a trajetória do Programa pode ser considerada vitoriosa para a comunidade do $\mathrm{CHC}$, para os departamentos de ensino da UFPR, para os 372 formados, para os próximos que virão e para a sociedade como um todo. Essa, em última análise, é a maior beneficiária do PRIMAH, já que recebe anualmente cerca de 60 novos especialistas capacitados para trabalhar em equipe e orientados pelas diretrizes do SUS para atender às necessidades de saúde da população.

É importante que se fomentem iniciativas de abertura de novos programas de residência multiprofissional, bem como de fortalecimento e consolidação dos programas já existentes, para que os benefícios aqui apresentados possam ser ampliados com a transformação das práticas profissionais, o desenvolvimento de competências do trabalho em equipe, a efetiva integração ensino-serviço e avanços na formação de profissionais de excelência.

São necessários mais estudos que apresentem os desafios da RMS no país e discutam a relação entre o ensino teórico e a prática profissional, a estruturação da gestão da pesquisa nos serviços e o fortalecimento da formação em saúde.

\section{REFERÊNCIAS}

1. Brasil. Presidência da República. Lei n 11.129, de 30 de junho de 2005. Institui o Programa Nacional de Inclusão de Jovens - ProJovem, cria o Conselho Nacional da Juventude - CNJ e a Secretaria Nacional de Juventude, altera as Leis $n^{\circ}$ s 10.683, de 28 de maio de 2003, e 10.429, de 24 de abril de 2002, e dá outras providências. [acesso em 23 out 2019]. Disponível em: http://portal.saude.gov.br/portal/arquivos/ pdf/Lei\%20n11129 05.pdf.

2. Ministério da Educação (Brasil). Comissão Nacional de Residência Multiprofissional em Saúde. Resolução $n^{\circ} .2$, de 13 de abril de 2012. Diretrizes Gerais para os Programas de Residência Multiprofissional e em Profissional de saúde. Diário Oficial da União 16 abr 2012; Seção I [acesso em 10 out 2019]. Disponível em: http://portal.mec.gov.br/index.php?option=com docman\&view=download\&alias=15448-resol-cnrms-n2-13abril-2012\&ltemid=30192.

3. Nascimento DDG, Oliveira MAC. Competências profissionais e o processo de formação na residência multiprofissional em Saúde da Família. Saúde Soc. [Internet]. 2010 [acesso em 8 de nov 2019]; 19(4):814827. Disponível em: http://dx.doi.org/10.1590/S0104-12902010000400009.

4. Salvador AS, Medeiros CS, Cavalcanti PB, Carvalho RN. Construindo a Multiprofissionalidade: um olhar sobre a residência multiprofissional em saúde da família e comunidade. Rev. bras. ciênc. Saúde [Internet]. 2011[acesso em 8 de nov 2019]; 15(3):329-338. Disponível em: https://pesquisa.bvsalud.org/portal/ resource/pt/lil-613382.

5. Ministério da Saúde (Brasil). Secretaria de Gestão do Trabalho e da Educação na Saúde. Departamento de Gestão da Educação na Saúde. Residência multiprofissional em saúde: experiências, avanços e desafios. Brasília: 2006 [acesso em 8 de nov 2019]. Disponível em: http://bvsms.saude.gov.br/bvs/ publicacoes/residencia multiprofissional.pdf.

6. Albuquerque VS, Gomes AP, Rezende CHA, Sampaio MXS, Dias OV, Lugarinho RM. A integração ensino-serviço no contexto dos processos de mudança na formação superior dos profissionais da saúde. RBEM [Internet]. 2008;32(3):356-362 [acesso em 20 de julho de 2020]. Disponível em: http://www.scielo. br/pdf/rbem/v32n3/v32n3a10.pdf. 


\title{
DEZ ANOS DA RESIDÊNCIA MULTIPROFISSIONAL DO COMPLEXO HOSPITAL DE CLIINICAS DA UNIVERSIDADE FEDERAL DO PARANÁ
}

\section{RESUMO:}

Objetivo: relatar a experiência dos dez anos de implementação do Programa de Residência Multiprofissional em Atenção Hospitalar no Complexo Hospital de Clínicas da Universidade Federal do Paraná. Desenvolvimento: relato de experiência, sobre os dez anos de implementação do programa de residência. Os dados foram coletados de documentos internos e dos relatos dos coordenadores e preceptores. Trezentos e setenta e dois profissionais foram formados. Dentre os avanços do programa, destaca-se o aumento no número de vagas e especialidades, consolidação do Regimentos Interno, gerenciamento informatizado das informações Acadêmicas, incentivo à pesquisa e melhoria da assistência no âmbito hospitalar. Considerações finais: Os dez anos de programa foram marcados pelo desafio da desconstrução do olhar uniprofissional para um projeto que contemplasse a diversidade das múltiplas profissões, proporcionando a construção de novos paradigmas, a transformação das práticas profissionais, o desenvolvimento de competências do trabalho em equipe, a integração ensino-serviço e o avanço na formação.

DESCRITORES: Educação em Enfermagegm; Educação em Saúde; Internato e Residência; Internato não Médico.

\section{DIEZ AÑOS DE LA RESIDENCIA MULTIPROFESIONAL EN EL COMPLEJO HOSPITAL DE CLÍNICAS DE LA UNIVERSIDAD FEDERAL DE PARANÁ}

\section{RESUMEN:}

Objetivo: relatar la experiencia de los diez años de implementación del Programa de Residencia Multiprofesional en Atención Hospitalaria en el Complejo Hospital de Clínicas de la Universidad Federal de Paraná. Desarrollo: reporte de experiencia de los diez años de implementación del programa de residencia. Los datos se obtuvieron de documentos internos e informes de coordinadores y tutores. Se capacitó a trescientos setenta y dos profesionales. Entre los avances del programa, cabe destacar el incremento en el número de vacantes y especialidades, la consolidación del Reglamento Interno, la gestión computarizada de la información académica, el incentivo a la investigación y el mejoramiento de la asistencia en el ámbito hospitalario. Consideraciones finales: los diez años del programa estuvieron marcados por el desafío de deconstruir la mirada uniprofesional de un proyecto que contempló la diversidad de múltiples profesiones, propiciando la construcción de nuevos paradigmas, la transformación de prácticas profesionales, el desarrollo de habilidades de trabajo en equipo, la integración enseñanza-trabajo y avance en la formación.

DESCRIPTORES: Educación en Enfermería; Educación en Salud; Internado y Residencia; Internado no Médico.

Recebido em: 25/08/2020

Aprovado em: 29/03/2021

Editora associada: Luciana Puchalski Kalinke

\author{
Autor Correspondente: \\ Liane Fuhr Pivatto \\ Complexo Hospital de Clínicas da Universidade Federal do Paraná - Curitiba, PR, Brasil \\ E-mail: liane_hc@hotmail.com
}

\begin{abstract}
Contribuição dos autores:
Contribuições substanciais para a concepção ou desenho do estudo; ou a aquisição, análise ou interpretação de dados do estudo - Andrzejevski VMS, Pereira RAB; Elaboração e revisão crítica do conteúdo intelectual do estudo - Brusamarello T; Responsável por todos os aspectos do estudo, assegurando as questões de precisão ou integridade de qualquer parte do estudo - Pivatto LF, Félix JVC. Todos os autores aprovaram a versão final do texto.
\end{abstract}

Copyright ( 2021 Este é um artigo em acesso aberto distribuído nos termos da Licença Creative Commons Atribuição, que permite o uso irrestrito, a distribuição e reprodução em qualquer meio desde que o artigo original seja devidamente citado. 